\title{
Efectos de un programa de actividad física en la calidad del sueño de escolares con TDAH
}

\section{Effects of a physical activity program on the sleep quality of schoolchildren with ADHD}

\author{
López Sánchez, G.F.*, López Sánchez, L. y Díaz Suárez, A. \\ Facultad de Ciencias del Deporte, Universidad de Murcia, España
}

\begin{abstract}
Resumen: Este artículo se centra en estudiar los efectos de un programa de actividad física sobre la calidad del sueńo de un grupo de escolares con Trastorno de Déficit de Atención con Hiperactividad (TDAH). Han participado 12 escolares (12 niños), entre los 7 y los 12 años de edad. La calidad del sueño se ha medido mediante el Índice de Calidad del Sueño de Pittsburg. El procedimiento ha sido: pre-test, intervención y pos-test. La intervención ha consistido en 2 días a la semana de actividad física, 60 minutos al día, durante 12 semanas. Se ha producido una mejora de la calidad del sueńo en un $16.6 \%$ de los escolares. Tras la intervención algunos escolares han mantenido su nivel de calidad del sueńo y otros lo han aumentado.

Palabras clave: Calidad del Sueño, Actividad Física, Salud, Educación Primaria, TDAH.
\end{abstract}

Abstract: this paper focuses on studying the effects of a physical activity program on sleep quality of a group of schoolchildren with Attention Deficit Hyperactivity Disorder (ADHD). This investigation involved 12 students (12 boys), aged between 7 and 12 years. The sleep quality was measured by Pittsburg's Sleep Quality Index. The procedure was as follows: pre-test, intervention and post-test. The intervention consisted of 2 days per week of physical activity, 60 minutes per day, during 12 weeks. 16.6 $\%$ of schoolchildren have increased their sleep quality. After the physical activity program, some schoolchildren have maintained their sleep quality and others have increased it.

Keywords: Sleep Quality, Physical Activity, Health, Elementary Education, ADHD.

\section{Introducción}

El trastorno de déficit de atención con/sin hiperactividad (TDAH) es, junto con el asma y la obesidad, una de las enfermedades más prevalentes durante la infancia, tal y como indican López-Sánchez, López-Sánchez \& Díaz-Suárez (2015a). Ya en 1993, Michanie, Márquez, Estévez \& Steimberg, afirmaban que aún no se disponía de un tratamiento curativo para el TDAH, pero ya proponían una serie de medidas terapéuticas eficaces para reducir las manifestaciones, consistentes en la combinación de un abordaje farmacológico (mediante estimulantes) y distintas intervenciones psicoterapéuticas (técnicas de orientación y entrenamiento a padres, de tipo cognitivo-conductual, con el objetivo disminuir el estrés que estas familias suelen presentar).

El interés por encontrar tratamientos que sean eficaces para reducir el TDAH ha ido aumentando a lo largo del tiempo (Barkley, 1997) y se han realizado diversas aportaciones. Existen tratamientos, entre los que se incluyen el psicológico, el psiquiátrico, el farmacológico y la modificación de conducta (García García et al, 2008). Asimismo, García-Chávez \& Hernández-Vicente (2009) distinguen dos tipos de intervención: tratamiento farmacológico e intervención conductual, mientras que Sances-Masero (2009) habla de tres modalidades de intervención del TDAH: farmacológica, psicosocial

Dirección para correspondencia [Correspodence address]: Guillermo Felipe López Sánchez. E-mail: gfls@um.es y combinada (terapia farmacológica y psicosocial). Según Grau-Sevilla (2007), los nińos con TDAH están recibiendo tratamiento en un $97.4 \%$ de los casos, de los cuales en un $64 \%$ de los casos reciben tratamiento combinado (tanto psicológico como farmacológico), seguido del tratamiento sólo farmacológico en un $20.2 \%$ de los casos y sólo psicológico en un $13.2 \%$. Un dato a destacar por tanto es que el $84.2 \%$ de los niños con TDAH recibe medicación.

Félix-Mateo (2006) señala la conveniencia de combinar, junto con la intervención psicopedagógica, el tratamiento farmacológico. En la misma línea, Bitaubé et al. (2009) indican que el tratamiento debe incorporar medidas farmacológicas y psicosociales. Según Ureńa-Morales (2007), el tratamiento que ha demostrado mayor efectividad es el "multidisciplinar" que combina los siguientes tratamientos: tratamiento psicológico, tratamiento farmacológico y tratamiento psicopedagógico. Igualmente, Herranz \& Argumosa (2000) precisan que se debe establecer un tratamiento multidisciplinario del nińo: apoyo psicopedagógico, información exhaustiva y tratamiento farmacológico. También Rubió-Badía et al. (2006) y Jarque-Fernández (2012) indicaron que el tratamiento en muchos casos ha de ser un tratamiento multimodal y contextualizado. Siguiendo a Millán-Lara (2009), esta intervención multimodal debe incluir psicoeducación y entrenamiento de padres, intervenciones psicológicas-conductuales o cognitivo-conductuales, intervenciones escolares y psicopedagógicas 
y si fuese necesario tratamiento farmacológico. Para Lora Espinosa (2006), los pilares del tratamiento son: plan de acción, educación, tratamiento farmacológico, no farmacológico y revisiones periódicas.

Con respecto al tratamiento farmacológico, el tratamiento de elección es el metilfenidato por su eficacia, seguridad y coste-efectividad (García-García et al., 2008; Lora-Espinosa, 2006). En cuanto al tratamiento farmacológico diferente al metilfenidato, el fármaco no estimulante que más datos científicos tiene apoyando su eficacia y seguridad en nińos y adolescentes con TDAH es la atomoxetina, y es el único indicado como de primera elección, junto con los estimulantes (Díez et al., 2006).

Respecto a los tratamientos no farmacológicos, en los últimos tiempos han aparecido nuevas líneas de interés, más allá de los habituales tratamientos conductuales o cognitivoconductuales (Cardo \& Servera, 2008). Entre ellas, Cidoncha (2010) destaca el papel favorable de la Educación Física, debido a que permite trabajar la inhibición muscular, el control postural, la relajación y la autoestima, tan fundamental para ellos, ya que les beneficia en su rendimiento académico, sus relaciones sociales y su autoconocimiento. Rosal-Giménez (2008) también propone una serie de actividades para tratar de facilitar en el niño/a con TDAH la relajación, el autocontrol, la atención, la concentración y la reducción de la tensión, entre las que incluye ejercicios de saltos, levantamientos de pesos y baile. Además se pueden proponer situaciones de resolución de problemas como estrategia de trabajo con niños diagnosticados con TDAH (Ochoa-Angrino et al., 2006). También son interesantes las intervenciones no farmacológicas del TDAH en el hogar o entorno familiar, las cuales incluyen informar a los padres sobre los diferentes aspectos del trastorno y de cómo pueden afectar a su hijo, e instruirles en nociones de terapia del comportamiento y terapia cognitiva para un mejor control de la conducta perturbadora, de la falta de organización y atención del niño (Eddy-Ives, 2006).

Una detección y tratamiento precoces ayudarán a controlar los síntomas, mejorando el aprendizaje escolar y las interacciones sociales del TDAH (García-García et al., 2008; Martínez de Haro et al., 2003). Destaca la situación actual, en la cual las familias, los profesores y orientadores psicopedagógicos, desconocedores de la naturaleza de este problema, de sus características y de las alternativas de tratamiento, se sienten incapaces de ofrecer ayuda adecuada a estos nińos e incluso les malinterpretan en su conducta, procediendo a una cierta marginación y a la consideración de niños malos, revoltosos, rebeldes, etc. (San Sebastián, 2005). Es por ello de suma importancia desarrollar estrategias de formación (Gue-
rrero-López \& Pérez-Galán, 2011; Herranz-Jordán, 2006) y que haya una adecuada cooperación entre el pediatra y el maestro, entre la escuela y el centro de salud (RodríguezSalinas Pérez et al., 2006). Además, se deben tener en cuenta una serie de orientaciones educativas para niños con TDAH, como las propuestas por Cortés Ariza (2010), entre las que se pueden destacar la coordinación profesores-equipo de orientación-familias, estrategias de afrontamiento y resolución de problemas, disciplina y buena relación afectiva, dar la oportunidad de desarrollar lo que saben hacer bien, no realizar tareas largas y complejas, técnicas de modificación de conducta: elogiar, recompensar y establecer límites.

Otro aspecto importante, es que el TDAH en ocasiones aparece asociado a problemas en diferentes parámetros saludables como condición física, composición corporal, imagen corporal, frecuencia cardiaca, tensión arterial, saturación de oxígeno en sangre, coordinación dinámica general y segmentaria, calidad de vida, o calidad del sueño (López-Sánchez, López-Sánchez \& Díaz Suárez, 2014, 2015 a, b, c, d, 2016 a, b). En concreto, el presente estudio se centra en la calidad del sueño, la cual ha sido asociada con el TDAH en estudios como los de Schredl et al (2007), Fajardo (2008) y Konikowska et al (2012).

Por todo ello, es necesaria la realización de más investigaciones sobre estas cuestiones para poder precisar mejor sus implicaciones y elaborar adecuadas estrategias para la prevención y el tratamiento del TDAH y de sus complicaciones. Nuestra propuesta plantea, por tanto, un tratamiento no farmacológico innovador, basado en la realización de actividad física de forma regular (dos días a la semana), el cual tiene como objetivo mejorar la salud, y en concreto la calidad del sueño, de los niños y adolescentes con TDAH.

\section{Método}

\section{Características de los participantes y muestra}

La muestra inicial estaba compuesta por 18 escolares de sexo masculino de la Región de Murcia, con una media de edad de 10.05 ańos (DT 1.80) y un rango de edad de 7 a 14 ańos. Estos 18 escolares completaron el pretest pero, durante la investigación, 6 de los escolares tuvieron que abandonar el estudio. Por tanto, la muestra final quedó reducida a 12 escolares de sexo masculino, con una media de edad de 9.83 años (DT 1.51) y un rango de edad de 7 a 12 ańos. Estos 12 escolares han completado el pretest, la intervención y el postest. En la Tabla 1 se describe la muestra. 
Tabla 1. Descripción de la muestra.

\begin{tabular}{ll}
\hline \multicolumn{2}{l}{ SUJETOS QUE COMPLETARON PRETEST } \\
\hline Número de sujetos & 18 \\
Media de edad & 10.05 (DT 1.80) \\
Rango de edad & $7-14$ \\
Sexo Masculino & 18 \\
$\quad$ Femenino & 0 \\
\hline SUJETOS QUE COMPLETARON PRETEST, INTERVEN- \\
CIÓN Y POSTEST \\
Número de sujetos \\
Media de edad \\
Rango de edad & 9.83 (DT 1.51) \\
Sexo Masculino $\quad 12$ \\
$\quad$ Femenino & 0 \\
\hline
\end{tabular}

Esta investigación ha sido aprobada por la Comisión de Ética de Investigación de la Universidad de Murcia y los padres/ tutores de todos los participantes han firmado un consentimiento informado.

\section{Diseño y variables de estudio}

El diseño ha sido un diseño cuasiexperimental pre-post con un solo grupo de intervención ya que el alumnado fue seleccionado por presentar el trastorno, de forma no aleatoria, y se consideró como criterio de inclusión de la investigación que todos los alumnos con TDAH recibieran tratamiento.

Los sujetos participantes en el estudio han realizado un pre-test, una intervención de 12 semanas y un pos-test.

En el pre-test se ha analizado la variable Calidad del Sueño.

Durante la intervención, los escolares participantes han realizado sesiones de actividad física dos días a la semana (60 minutos cada día) fuera del horario lectivo, en las que los alumnos han llevado a cabo circuitos y ejercicios destinados a mejorar su condición física, especialmente la inhibición muscular y el control postural, enfatizando en la relajación y en la autoestima. La actividad física ha sido aeróbica y de intensidad media-alta. Las sesiones han sido dirigidas por el personal investigador.

En el pos-test se ha vuelto a analizar la Calidad del Sueño de los escolares para comprobar si se han producido mejoras respecto al pre-test.

\section{Procedimiento}

Tras la revisión de la literatura, se han mantenido diferentes reuniones entre el personal investigador para organizar la obtención de datos y revisar los protocolos de medición. Además se han realizado reuniones con los responsables de una
Asociación de Ayuda al Déficit de Atención con más o menos Hiperactividad y con los padres de los sujetos de la muestra, para concretar las fechas y horarios del pretest, la intervención y el postest. Igualmente, se han llevado a cabo reuniones periódicas con el objetivo de realizar un seguimiento sistemático del trabajo realizado.

También se hizo entrega a los padres de un modelo de consentimiento informado en donde se detallan las pruebas y objetivos de la investigación. Tras el visto bueno paterno, los investigadores comenzaron con la recogida de datos y la intervención.

El enfoque metodológico en la intervención se ha llevado a cabo desde una perspectiva fundamentalmente lúdica. Contando con la motivación natural de los alumnos hacia el juego y la actividad deportiva y conduciéndola hacia la cooperación, el respeto a las reglas, el esfuerzo por superar las dificultades, la autonomía y la alegría por la tarea bien hecha.

\section{Análisis de variables}

Se ha evaluado la calidad del sueño, ya que los problemas y trastornos en el sueño suelen ser comunes en el TDAH (Schredl et al., 2007; Fajardo, 2008; Konikowska et al., 2012). El Índice de Calidad del Sueño de Pittsburg proporciona una puntuación global de la calidad del sueño y puntuaciones parciales en siete componentes distintos: calidad subjetiva del sueño, latencia, duración, eficiencia habitual, alteraciones, uso de medicación hipnótica y disfunción diurna. Las preguntas hacen referencia al último mes. La puntuación de cada uno de los siete componentes oscila entre 0 (no existe dificultad) y 3 (grave dificultad); la puntuación global tiene un rango entre 0 (ninguna dificultad) y 21 (dificultades en todas las áreas), con un punto de corte en la puntuación 5 para diferenciar a los buenos de los malos dormidores (Buysse et al., 1989; Sierra et al., 2002).

\section{Análisis de datos}

Se ha realizado un análisis estadístico por medio del Statistical Package for Social Sciences 22.0 (SPSS-22.0). Se han seguido las indicaciones del Manual de estadística aplicada a las Ciencias de la Actividad Física y el Deporte (Ortega, Ortiz \& Artés, 2009).

\section{Resultados}

A continuación se presentan los resultados en dos tablas:

La tabla 2 es un resumen de la prevalencia de buenos y malos dormidores. Para elaborar esta tabla se ha tomado en consideración la muestra inicial $(\mathrm{n}=18)$ y se han analizado los resultados de los sujetos en el pretest. 
Tabla 2. Calidad del Sueńo de la muestra inicial, $\mathbf{N}=18$

\begin{tabular}{lc}
\hline Variable & Porcentajes \\
\hline \multirow{2}{*}{ Calidad del Sueño } & Buen dormidor: $72,2 \%$ \\
& Mal dormidor: $27,8 \%$ \\
\hline
\end{tabular}

La tabla 3 muestra la comparación de prevalencia de buenos y malos dormidores entre el pretest y el postest. Para elaborar esta tabla se ha tomado en consideración la muestra final $(\mathrm{n}=12)$.

Tabla 3. Comparación Calidad del Sueño Pre-Post de la muestra final, N=12.

\begin{tabular}{lccc}
\hline Variable & $\%$ Pretest & $\%$ Postest & Diferencia \\
\hline & Buen dormidor: & Buen dormidor: & Buen dormidor: \\
Calidad del Sueño & $66.7 \%$ & $83.3 \%$ & $+16.6 \%$ \\
& Mal dormidor: & Mal dormidor: & Mal dormidor: \\
& $33.3 \%$ & $16.7 \%$ & $-16.6 \%$ \\
\hline
\end{tabular}

\section{Discusión}

Al analizar la calidad del sueño y las prevalencias de buenos y malos dormidores, se ha encontrado que los problemas y trastornos en el sueño son comunes en el TDAH, como ya habían señalado algunos autores (Schredl et al., 2007; Fajardo, 2008; Konikowska et al., 2012), ya que casi un tercio de los niños de la muestra presenta problemas y trastornos en el sueño.

En cuanto a los efectos de la intervención, los resultados son más difíciles de discutir debido a la escasez de investigaciones que han intervenido sobre estos parámetros en niños con TDAH. No obstante, se puede señalar que la prevalencia de buenos dormidores ha aumentado tras el programa de actividad física, es decir la calidad del sueño ha aumentado.

Los resultados de esta investigación se pueden comparar con otros estudios de la literatura científica, que a pesar de no intervenir directamente sobre la calidad del sueño, sí que realizan intervenciones sobre niños con TDAH para intentar mitigar los síntomas del trastorno y consiguen resultados positivos. Se pueden encontrar diferentes tipos de intervenciones como la de Fernández-Martín et al. (2003), que llevan a cabo una intervención cognitivo-conductual basada en la formación de padres, docentes y alumnado, la cual consigue producir mejoras significativas en las conductas problema que presentaban los alumnos en el contexto escolar. Otra propuesta de intervención educativa es la de Escalera-García (2009), que interviene con el alumno, con sus compañeros y con sus padres, mediante un tratamiento cognitivo-conductual (técnica de ganancia de puntos, uso de reforzadores, actividades de relajación muscular y actividades de valores y conductas). O el programa de intervención multidisciplinar que plantea Ruiz-Triviño (2010), consistente en la formación de tutores, familiares y profesorado y en la realización de actividades encaminadas a la potenciación de la atención, las habilidades sociales y el autocontrol de los alumnos. Es también destacable la intervención llevada a cabo por Presentación-Herrero et al. (2010) con nińos con TDAH, sus padres y sus profesores, la cual incluyó modificación de conducta, técnicas cognitivo-conductuales, adaptaciones académicas y habilidades sociales y consiguió mejoras duraderas tras el tratamiento, especialmente en las áreas académica y social.

Entre las intervenciones, ocupan un papel destacado las intervenciones mediante actividad física. Gapin et al. (2011) revisan la evidencia existente sobre los efectos de la actividad física en los síntomas del TDAH y señalan que la actividad física puede beneficiar los síntomas de comportamiento y el rendimiento cognitivo de los nińos con TDAH, por lo que podría ser un complemento eficaz a la medicación o un tratamiento alternativo para aquellos nińos que no responden a los tratamientos de medicación o desean buscar formas alternativas de tratamiento. Esta es también la hipótesis de Wigal et al. (2013) que plantean que el ejercicio físico altera la fisiología subyacente presente en el TDAH y podría constituir una importante alternativa y/o un tratamiento complementario al farmacológico. En esta línea, Mena-Rodríguez et al. (2008), aplicaron una estrategia pedagógica basada en la psicomotricidad a través de muchas actividades como el baile y el juego y encontraron que los nińos con TDAH trabajaban mejor y disfrutaban más de las actividades académicas en la escuela, a la vez que reducían sus niveles de hiperactividad y de déficit de atención. Otro estudio similar es el de Azrin et al. (2007), sobre el uso de la actividad física como refuerzo para la tranquilidad de los niños con TDAH en el aula, que mostró un aumento de la calma en los nińos durante la duración de la clase. O el de Barnard-Brak et al. (2011), que establecieron una asociación entre la Educación Física y los síntomas del TDAH, sugiriendo que la Educación Física, como una forma estructurada de la actividad física, se puede considerar asociada con menores niveles de los síntomas del TDAH a lo largo del tiempo.

Otro tipo de intervención mediante actividad física que ha mostrado resultados positivos es la de los campamentos de verano. Así, Gerber-von Muller et al. (2009), en un campamento de verano intensivo con nińos y adolescentes con TDAH, desarrollaron y evaluaron un programa de inter- 
vención multimodal (entrenamiento de habilidades sociales, actividades deportivas y medicación), consiguiendo mejoras significativas de larga duración en los síntomas del TDAH tras el campamento de verano. Igualmente, Gerber et al. (2012) aplicaron un tratamiento multimodal (metilfenidato, entrenamiento de habilidades sociales, entrenamiento de la atención y participación en deportes), en el formato de un campamento de verano intensivo, y obtuvieron mejoras duraderas en las funciones neuropsicológicas de los niños y adolescentes con TDAH. De forma similar, Yamashita et al. (2011) llevaron a cabo un programa multidisciplinar de tratamiento de verano, en el cual se incluyó entrenamiento en habilidades deportivas, sociales y académicas, en niños con TDAH y los resultados mostraron que la mayoría de niños consiguió cambios positivos en el comportamiento y en algunas funciones cognitivas. Por último, Hupp et al. (2002), mediante un programa de verano, mostraron que el uso de recompensas y alabanzas puede mejorar la conducta deportiva de los nińos con TDAH.

Son también relevantes otras investigaciones como la de Tantillo et al. (2001) que evaluaron los efectos del ejercicio en nińos con TDAH, obteniendo resultados positivos que alientan a realizar nuevos estudios con el fin de corroborar si una sesión de ejercicio vigoroso tiene eficacia en la gestión del comportamiento del TDAH. O la de Kang et al. (2011), que llevaron a cabo una terapia mediante deporte en niños con TDAH, cuyos resultados demostraron una correlación positiva entre el deporte y la mejora de los síntomas de la atención, los síntomas cognitivos y las habilidades sociales, por lo que los autores del estudio sugirieron que la terapia mediante deporte puede aliviar los síntomas de atención y aumentar la competencia social en los niños con TDAH. En la misma línea, Smith et al. (2013) llevaron a cabo una intervención mediante actividad física en niños con TDAH y los resultados mostraron que la mayoría de los participantes mostraron mejoría general después del programa, por lo que los autores sugieren que la actividad física se muestra prometedora para tratar los síntomas del TDAH. Kiluk et al. (2009) también sugirieron que la participación activa en deportes puede estar asociada a menores niveles de ansiedad o depresión en niños con TDAH. Asimismo, en el programa terapéutico basado en la práctica deportiva de Lufi \& Parish-Plass (2011), llevado a cabo durante un año académico, se produjeron mejoras en el comportamiento y se redujo la ansiedad de los niños con TDAH.

Otros autores que han contribuido a la investigación en este campo son Patel \& Curtis (2007), los cuales realizaron un tratamiento multidimensional (nutrición, control del ambiente y terapia comportamental, educacional, física y del lenguaje) en niños con TDAH y documentaron que todos los niños mostraron mejoras significativas en las áreas de interacción social, concentración, escritura, lenguaje y comportamiento. Igualmente, Pontifex et al. (2013) mostraron que el ejercicio mejora el comportamiento, la atención y la actuación académica en niños con TDAH, de tal forma que sesiones individuales de ejercicio aeróbico de intensidad moderada pueden tener implicaciones positivas en la función neurocognitiva y el control inhibitorio en niños con TDAH. Además parece ser que las actividades al aire libre reducen los síntomas del TDAH (Kuo \& Faber Taylor, 2004), como la equitación (Rubio Jiménez \& García Gómez, 2011). Por último, cabe destacar el estudio de Medina et al. (2010), en el que midieron el impacto de la actividad física de alta intensidad en la atención sostenida niños diagnosticados con TDAH y encontraron que los déficits de atención de los niños pueden ser minimizados a través de actividad física, aunque se necesitan más estudios que confirmen que el ejercicio mitiga los síntomas del TDAH.

Por tanto, a modo de síntesis, el presente estudio plantea también una intervención mediante actividad física, pero realiza una innovadora aportación en relación a la literatura previa sobre actividad física y TDAH: La intervención no se centra sólo en mitigar los principales síntomas del TDAH (inatención y/o hiperactividad-impulsividad), sino que también intenta mejorar otra área donde los niños con TDAH suelen presentar problemas y que ha sido menos estudiada hasta el momento. Esta área es la calidad del sueño.

\section{Conclusiones}

Los niños con TDAH pueden presentar problemas en el sueño y una menor calidad del sueño.

Una intervención mediante actividad física de intensidad media-alta, con dos sesiones por semana de 60 minutos cada una, puede producir mejoras en la calidad del sueńo de niños con TDAH.

Se recomienda llevar a cabo otros programas de intervención mediante actividad física, de diferente duración y con diferentes metodologías, y medir los efectos de los mismos sobre la salud de los niños con TDAH, de tal forma que haya más recursos disponibles para reducir los síntomas de este trastorno y mejorar los diferentes parámetros saludables de los niños con TDAH.

Financiación: Fundación Séneca - Agencia de Ciencia y Tecnología de la Región de Murcia. Consejería de Industria, Turismo, Empresa e Innovación de la CARM. 


\section{Referencias bibliográficas}

1. Azrin, N. H., Vinas, V. \& Ehle, C. T. (2007). Physical activity as reinforcement for classroom calmness of ADHD children: A preliminary study. Child \& Family Behavior Therapy, 29 (2), 1-8.

2. Barkley, R. (1997). ADHD and the nature of self-control. New York: Guilford Press.

3. Barnard-Brak, L., Davis, T., Sulak, T. \& Brak, V. (2011). The Association Between Physical Education and Symptoms of Attention Deficit Hyperactivity Disorder. Journal of Physical Activity \& Health, 8 (7), 964-970.

4. Bitaubé, J. A., López-Martín, S., Fernández-Jaén, A. \& Carretié Arangüena, L. (2009). Trastorno por déficit de atención con o sin hiperactividad. Jano, 1729, 18-26.

5. Buysse D. J., Reynolds C. F., Monk T. H., Berman S. R. \& Kupfer D. J. (1989). The Pittsburgh Sleep Quality Index: A new instrument for psychiatric practice and research. Psychiatry Research, 28 (2), 193-213.

6. Cardo, E. \& Servera, M. (2008). Trastorno por déficit de atención/hiperactividad: Estado de la cuestión y futuras líneas de investigación. Revista de Neurología, 46 (6), 365-372.

7. Cidoncha Delgado, A. I. (2010). Niños con Déficit de Atención por Hiperactividad TDAH: Una Realidad Social en el Aula. Revista Autodidacta, 1 (4), 31-36.

8. Cortés Ariza, M. C. (2010). Déficit de Atención con Hiperactividad (TDAH): Concepto, Características e Intervención Educativa. Innovación y Experiencias Educativas, 28, 1-8.

9. Díez Suárez, A., Figueroa Quintana, A. \& Soutullo Esperón, C. (2006). Trastorno por déficit de atención e hiperactividad (TDAH): comorbilidad psiquiátrica y tratamiento farmacológico alternativo al metilfenidato. Revista Pediatría de Atención Primaria, 8 (4), 135-155.

10. Eddy Ives, L. (2006). Intervenciones no farmacológicas en el entorno familiar de niños con trastorno por déficit de atención con/sin hiperactividad. Revista Pediatría de Atención Primaria, 8 (4), 57-67.

11. Escalera García, M. R. (2009). Una rentable propuesta de intervención educativa para el niño con TDAH. Innovación y Experiencias Educativas, 16, 1-10.

12. Fajardo, A. (2008). Trastorno deficitario de atención e hiperactividad y sueño. Acta Neurológica Colombiana, 24, 1, S64-S72.

13. Félix Mateo, V. (2006). Recursos para el diagnóstico psicopedagógico del TDAH y comorbilidades. Revista Electrónica de Investigación Psicoeducativa, 10, 4 (3), 623-642.

14. Fernández Martín, F., Hinojo Lucena, F. J. \& Aznar Díaz, I. (2003). Dificultades del Alumnado con Trastorno por Déficit de Atención con Hiperactividad (TDAH) en el Aula: Implicaciones para la Formación Docente. Enseñanza, 21, 219-232.

15. Gapin, J. I., Labban, J. D. \& Etnier, J. L. (2011). The effects of physical activity on attention deficit hyperactivity disorder symptoms: The evidence. Preventive Medicine, 52 (1), S70-S74.

16. García Chávez, M. I. \& Hernández Vicente, E. I. (2009). Modificación de conceptos a padres y profesores en relación al TDAH. Tesis Doctoral. Universidad Michoacana de San Nicolás de Hidalgo.

17. García García, M. D., Prieto Tato, L. M., Santos Borbujo, J., Monzón Corral, L., Hernández Fabián, A. \& San Feliciano Martín, L. (2008). Trastorno por déficit de atención e hiperactividad: un problema actual. Anales de Pediatría, 69 (3), 244-250.

18. Gerber-von Mueller, G., Petermann, U., Petermann, F., Niederberger, U., Stephani, U., Siniatchkin, M. \& Gerber, W. D. (2009). ADHD summer camp: Development and evaluation of a multimodal intervention program. Kindheit und Entwicklung, 18 (3), 162-172.

19. Gerber, W. D., Gerber-von Mueller, G., Andrasik, F., Niederberger, U., Siniatchkin, M., Kowalski, J. T., Petermann, U. \& Petermann, F. (2012). The impact of a multimodal Summer Camp Training on neu- ropsychological functioning in children and adolescents with ADHD: An exploratory study. Child Neuropsychology, 18 (3), 242-255.

20. Grau Sevilla, M. D. (2007). Análisis del Contexto Familiar en Niños con TDAH. Tesis Doctoral. Universidad de Valencia.

21. Guerrero López, J. F. \& Pérez Galán, R. (2011). El alumnado con TDAH (hiperactividad) como colectivo en riesgo de exclusión social: propuestas de acción y de mejora. Revista RUEDES, 2, 37-59.

22. Herranz, J. L. \& Argumosa, A. (2000). Neuropediatría. Trastorno con déficit de atención e hiperactividad. Boletín de la Sociedad de Pediatría de Asturias, Cantabria, Castilla y León, 40 (172), 88-92.

23. Herranz Jordán, B. (2006). Trastorno por déficit de atención e hiperactividad: conocimientos y forma de proceder de los pediatras de Atención Primaria. Revista Pediatría de Atención Primaria, 8 (4), 217-239.

24. Hupp, S., Reitman, D., Northup, J., O’Callaghan, P \& LeBlanc, M. (2002). The Effects of Delayed Rewards, Tokens, and Stimulant Medication on Sportsmanlike Behavior With ADHD-Diagnosed Children. Behavior Modification, 26 (2), 148-162.

25. Jarque Fernández, S. (2012). Eficacia de las intervenciones con nińos y adolescentes con Trastorno por Déficit de Atención con Hiperactividad (TDAH). Anuario de Psicología, 42 (1), 19-33.

26. Kang, K. D., Choi, J. W., Kang, S. G. \& Han, D. H. (2011). Sports Therapy for Attention, Cognitions and Sociality. International Journal of Sports Medicine; 32, 953-959.

27. Kiluk, B. D., Weden, S. \& Culotta, V. P. (2009). Sport Participation and Anxiety in Children With ADHD. Journal of Attention Disorders, 12 (6), 499-506.

28. Konikowska, K., Regulska-Ilow, B. \& Rozanska, D. (2012). The influence of components of diet on the symptoms of ADHD in children. Roczniki Panstwowego Zakladu Higieny, 63, 2, 127-134.

29. Kuo, F. E., \& Faber Taylor, A. (2004). A Potential Natural Treatment for Attention-Deficit/Hyperactivity Disorder: Evidence From a National Study. American Journal of Public Health, 94 (9), 1580-1586.

30. López Sánchez, G. F., López Sánchez, L. \& Díaz Suárez, A. (2014). Effects of a physical activity program on the physical fitness of schoolchildren with ADHD. RICCAFD: Revista Iberoamericana de Ciencias de la Actividad Física y el Deporte, 3, 3, 24-37.

31. López Sánchez, G. F., López Sánchez, L. \& Díaz Suárez, A. (2015a). Trastorno por déficit de atención con hiperactividad (TDAH) y actividad física. EmásF: Revista Digital de Educación Física, 32, 1-13.

32. López Sánchez, G. F., López Sánchez, L. \& Díaz Suárez, A. (2015b). Effects of a physical activity program on the body composition of schoolchildren with ADHD. KRONOS: Revista Científica de Actividad Física y Deporte, 14, 2, 1-9.

33. López Sánchez, L., López Sánchez, G. F. \& Díaz Suárez, A. (2015c). Effects of a physical activity program on the body image of schoolchildren with ADHD. Cuadernos de Psicología del Deporte, 15, 2, 135-142.

34. López Sánchez, L., López Sánchez, G. F. \& Díaz Suárez, A. (2015d). Effects of a physical activity program on the heart rate, blood pressure and oxygen saturation of schoolchildren with ADHD. Actividad Física y Ciencias, 7, 1, 1-24.

35. López Sánchez, G. F., López Sánchez, L. \& Díaz Suárez, A. (2016a). Effects of a physical activity program on the general dynamic and segmentary coordination of boys with ADHD. Journal of Sport and Health Research (in press).

36. López Sánchez, G. F., López Sánchez, L. \& Díaz Suárez, A. (2016b). Effects of a physical activity program on the life quality of schoolchildren with ADHD. AGON: International Journal of Sport Sciences (in press).

37. Lora Espinosa, A. (2006). El tratamiento del niño y adolescente con TDAH en Atención Primaria desde el punto de vista de la evidencia. 
Revista Pediatría de Atención Primaria, 8 (4), 69-114.

38. Lufi, D. \& Parish-Plass, J. (2011). Sport-Based Group Therapy Program for Boys with ADHD or with Other Behavioral Disorders. Child \& Family Behavior Therapy, 33 (3), 217-230.

39. Martínez de Haro, V., Álvarez Barrios, M. J., Cid Yagüe, L., Garoz Puerta, I., Vega Marcos, R. de la \& Villagra Astudillo, A. (2003). Evaluación de la Salud en Educación Física. Educación Física y deporte escolar. Actas del V Congreso Internacional de FEADEF. Ed. AVAPEF. Valladolid, 313-317.

40. Medina, J. A., Netto, T. L., Muszkat, M., Medina, A. C., Botter, D., Orbetelli, R., Scaramuzza, L. F., Sinnes, E. G., Vilela, M. \& Miranda, M. C. (2010). Exercise impact on sustained attention of ADHD children, methylphenidate effects. Attention deficit and hyperactivity disorders, 2 (1), 49-58.

41. Mena Rodríguez, F. E., Salgado Muñoz, A. P. \& Tamayo Marín, P. A. (2008). Estrategia Pedagógica basada en la Lúdica y Psicomotricidad aplicada en las Áreas de Castellano y ciencias Naturales para Centrar la Atención de Niños y Niñas con TDAH de Preescolar y Básica Primaria de la Institución Educativa Ciudadela Cuba. Tesis de Licenciatura. Universidad Tecnológica de Pereira.

42. Michanie, C., Márquez, M., Estévez, P. \& Steimberg, L. (1993). Artículo especial. Trastorno por déficit de atención con hiperactividad (TDAH). Archivos Argentinos de Pediatría, 91, 1-12.

43. Millán Lara, L. (2009). El Alumno Adolescente con TDAH (Manual de Estrategias para Profesores de Educación Secundaria). Tesis de Licenciatura. Universidad Pedagógica Nacional. México, DF.

44. Ochoa Angrino, S., Cruz Panesso, I. \& Iván Valencia, A. (2006). Las situaciones de resolución de problemas como estrategia de trabajo con nińos diagnosticados con TDAH. Pensamiento Psicológico, 2 (7), 73-88.

45. Ortega, E., Ortiz, I. M. \& Artés, E. M. (2009). Manual de Estadística Aplicada a las Ciencias de la Actividad Física y el Deporte. Murcia: Diego Marín.

46. Patel, K. \& Curtis, L. T. (2007). A comprehensive approach to treating autism and attention-deficit hyperactivity disorder: A prepilot study. Journal of Alternative and Complementary Medicine, 13 (10), 10911097.

47. Pontifex, M. B., Saliba, B. J., Raine, L. B., Picchietti, D. L. \& Hillman, C. H. (2013). Exercise Improves Behavioral, Neurocognitive, and Scholastic Performance in Children with Attention-Deficit/Hyperactivity Disorder. The Journal of Pediatrics, 162 (3), 543-551.

48. Presentación, M. J., Siegenthaler, R., Jara, P. \& Miranda, A. (2010). Seguimiento de los efectos de una intervención psicosocial sobre la adaptación académica, emocional y social de nińos con TDAH. Psicothema, 22 (4), 778-783

49. Rodríguez-Salinas Pérez, E., Navas García, M., González Rodríguez, P., Fominaya Gutiérrez, S. \& Duelo Marcos, M. (2006). La escuela y el trastorno por déficit de atención con/sin hiperactividad (TDAH). Revista Pediatría de Atención Primaria, 8 (4), 175-198.

50. Rosal, I. M. (2008). Atención Educativa para el Alumnado con Trastorno por Déficit de Atención con Hiperactividad. Innovación y Experiencias Educativas, 9, 1-9.

51. Rubió Badía, I., Mena Pujol, B. \& Murillo Abril, B. (2006). El pediatra y la familia de un niño con TDAH. Revista Pediatría de Atención Primaria, 8 (4), 199-216.

52. Rubio Jiménez, J. C. \& García Gómez, A. (2011). Programa de iniciación a la equitación para alumnos con TEA y con TDAH. Consejería de Educación: Junta de Extremadura.

53. Ruiz Triviño, L. (2010). Programa para Alumnado con TDAH. Innovación y Experiencias Educativas, 27, 1-15.

54. San Sebastián, J. (2005). Trastorno por Déficit de Atención con Hiperactividad. Un trastorno del comportamiento precursor de otros. I Congreso Internacional de Trastornos del Comportamiento.

55. Sances Masero, C. (2009). Intervención Familiar Grupal en Niños con Trastorno por Déficit de Atención con Hiperactividad (TDAH). Tesis de Licenciatura. Universidad Abat Oliba CEU.

56. Schredl, M., Alm, B. \& Sobanski, E. (2007). Sleep quality in adult patients with attention deficit hyperactivity disorder (ADHD). European Archives of Psychiatry and Clinical Neuroscience, 257, 3, 164-168.

57. Sierra, J. C., Jiménez Navarro, C. \& Martín Ortiz, J. D. (2002). Calidad del Sueño en Estudiantes Universitarios: Importancia de la Higiene del Sueño. Salud Mental, 25, 6, 35-43.

58. Smith, A. L., Hoza, B, Linnea, K., McQuade, J. D., Tomb, M., Vaughn, A. J., Shoulberg, E. K. \& Hook, H. (2013). Pilot Physical Activity Intervention Reduces Severity of ADHD Symptoms in Young Children. Journal of Attention Disorders, 17 (1), 70-82.

59. Ureńa Morales, E. (2007). Guía Práctica con Recomendaciones de Actuación para los Centros Educativos en los Casos de Alumnos con TDAH. Islas Baleares: STILL. Asociación Balear de Padres de Niños con TDAH.

60. Tantillo, M., Kesick, C. M., Hynd, G. W., \& Dishman, R. K. (2001). The effects of exercise on children with attention-deficit hyperactivity disorder. Official Journal of the American College of Sports Medicine, 34, 2, 203-212.

61. Wigal, S. B., Emmerson, N., Gehricke, J. G. \& Galassetti, P. (2013). Exercise: Applications to Childhood ADHD. Journal of Attention Disorders, 17, 4, 279-290.

62. Yamashita, Y., Mukasa, A., Anai, C., Honda, Y., Kunisaki, C., Koutaki, J., Tada, Y., Egami, C., Kodama, N., Nakashima, M., Nagamitsu, S. \& Matsuishi, T. (2011). Summer treatment program for children with attention deficit hyperactivity disorder: Japanese experience in 5 years. Brain \& Development (Official Journal of the Japanese Society of Child Neurology), 33, 260-267. 